\title{
Electron Transport in Doped and Undoped Polymers Containing $\pi$-Conjugated Groups
}

\author{
Shogo SaIto, Tetsuo Tsutsui, Shizuo ToKito, \\ Takeo HARA, and Hsien-Tang CHIU \\ Department of Materials Science and Technology, \\ Graduate School of Engineering Sciences, \\ Kyushu University, Kasuga, Fukuoka 816, Japan
}

(Received August 20, 1984)

\begin{abstract}
Electric conduction behaviors were studied as for several polymers as a function of dopant concentration and the pyrolysis temperature. A weak electronic conduction is found to exist even in undoped states of poly ( $p$-phenylene sulfide), poly ( $m$-phenyleneisophthalamide) and poly(ethylene 2,6-naphthalate). The electronic conductivities of undoped poly( $p$-phenylene) and polythiophene are presumed to be very low. Stable semiconducting films were obtained from poly ( $p$-phenylene sulfide) doped with organic acceptors. The principal effect of introduction of the organic dopants is enhancement of the carrier mobility. A close relationship between the conductivity and the activation energy for conduction process is found in the doped poly $(p$ phenylene sulfide) family. From this relation, it is concluded that the conduction mechanism is based on the hopping one and metal-insulator transition does not occur in that doped with $\mathrm{AsF}_{5}$. Light pyrolysis of polyimide was a useful procedure for obtaining tough semiconducting films. Enhancement of the conductivity by pyrolysis is attributed to the development of the conjugated double bonds and closely connected with increase of the carrier mobility.

KEY WORDS Electronic Conductivity / Carrier Mobility / Doping /

Pyrolysis / Poly ( $p$-phenylene sulfide) / Poly ( $m$-phenyleneisophthalamide) /

Poly(ethylene 2,6-naphthalate) / Poly( $p$-phenylene) / Polythiophene /

Polyimide /
\end{abstract}

There are remaining problems in stability and durability of polyacetylene, though its electronic properties have been most extensively studied. Many other polymers have attracted much attention as a candidate of electron-conducting polymers which combined electronic conductivity, processibility and stability; poly ( $p$-phenylene sulfide), polypyrrole, pyrolyzed polyimide and so on. In the studies on the electrical properties of these stable polymeric films, much interests have been concentrated upon the highest value of attainable conductivity and less attention has been paid to conduction mechanism in the wide range of conductivity from insulator, semiconductor to metallic conductor.

In this paper, we will discuss the conduction mechanism in several stable polymers which contain $\pi$-conjugated groups as a function of dopant con- centration and chemical structure of polymer. Evaluation of carrier mobility in addition to the conductivity will throw light upon better understanding of the conduction mechanism in polymer films.

\section{EXPERIMENTAL}

Polymer used in this study are poly( $p$-phenylene sulfide) (PPS), poly ( $m$-phenylene isophthalamide) (PMIA), poly(ethylene 2,6-naphthalate) (PEN), poly ( $p$-phenylene) (PPP), polythiophene (PT), and $\operatorname{poly}\left(N, N^{\prime}\right.$-( $p, p^{\prime}$-oxyphenylene)pyromellitimide) (PI). Almost polymers were kindly supplied from the manufacturers. Samples for the electrical and optical measurements, except PPP, were films in shape whose thickness ranged from 20 to $125 \mu \mathrm{m}$. The sample of PPP was pellet prepared by com- 
pression of the powder.

For some samples, so-called doping was performed; PPS's were doped with organic acceptors in gas phase and PT's were doped with $\mathrm{ClO}_{4}{ }^{-}$by the electrochemical procedure. For some samples, heat treatment or the pyrolysis was performed in $\mathrm{N}_{2}$ atmosphere.

Conductivity measurements were carried out in the electrode arrangement of sandwich type for all samples; Electrode attached on both surfaces of the film and a guard electrode. Electrical conductivity was determined from the dark current measured with a conventional electrometer, for instance, a vibrating-reed electrometer. Drift mobility of electronic carriers was determined by the time-of-flight technique composed of a pulsed light source, a high speed amplifier and a digital storage oscilloscope.

\section{RESULTS AND DISCUSSION}

\section{Existence of Electronic Conduction in Undoped Polymers}

The temperature dependence of conductivity $\sigma$ in undoped PPS is shown in Figure 1. The experimental data can be described by two Arrhenius plots which intersect each other at around glass transition temperature $T_{\mathrm{g}}$. This result is similar to that observed in impurity-ion-conducting polymers, like poly(methyl methacrylate) or poly(vinyl chloride). However, the $\log \sigma-T^{-1}$ relations for PMIA and PEN in Figure 1 suggest that a hasty judgement for conduction mechanism in PPS should be avoided. The $\log \sigma-T^{-1}$ plots for PMIA or PEN break at temperature $T_{\mathrm{b}}$ which is entirely different from $T_{\mathrm{g}}$.

Figure 2 illustrates how the apparent conductivity of PPS depends on histories of both photoirradiation and thermal treatments. First, conductivity of the PPS without any particular pretreatment was measured with ascending temperature in dry air (curve 1). Next, the conductivity of the same film was measured with descending temperature, after it was annealed at $433 \mathrm{~K}$ which was far above $T_{\mathrm{g}}$ in a dark measuring chamber (curve 2). After this measurement, the $\log \sigma-T^{-1}$ relation in the annealed film (curve 2) was reproduced in a couple of succesive measurements in both ascending and descending temperature processes. Finally, the annealed film was irradiated for $5 \mathrm{~min}$ with a UVlight at room temperature. This procedure caused an increase of the apparent conductivity of the film

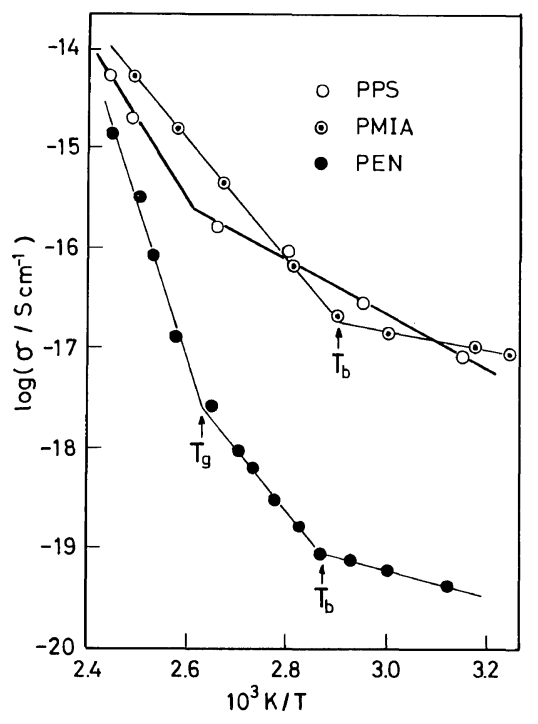

Figure 1. Temperatue dependences of the conductivity in undoped PPS, PMIA and PEN.

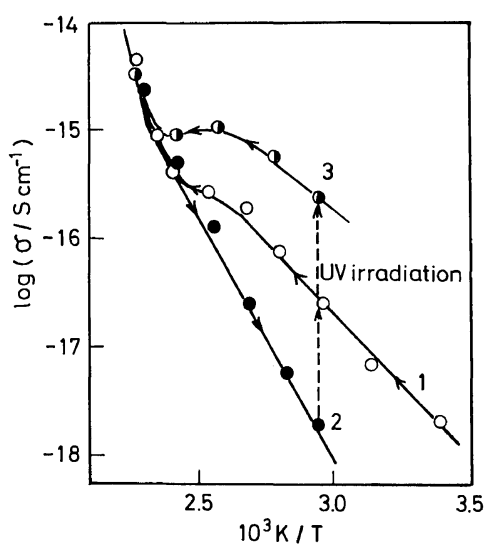

Figure 2. Temperature dependences of dark conductivity in PPS with different pre-irradiation and thermal histories.

by about three orders of magnitude. Curve 3 was obtained on this photo-irradiated film with ascending temperature. After the film had been annealed at $433 \mathrm{~K}$ again, it gave the same $\log \sigma-T^{-1}$ relation as curve 2 . In the preirradiated PPS which has not been annealed in dark, the dark currents continued to decrease for several hours or days after the application of electric field, and therefore, the value of $\sigma$ on curve 1 and 3 in Figure 2 are apparent one. These apparent conductivity should be attributed 
to thermal release of electronic carriers from deep trap sites.

One can recognize that the $\log \sigma-T^{-1}$ of curve 2 in Figure 2 should represent the intrinsic dark conductivity of the PPS film in dry air. This conductivity is also attributable to electronic conduction from the following two evidences. First, the conductivity was found to be very sensitive to oxygen atmosphere. When the conductivity of the annealed PPS was measured under high vacuum, the values of $\sigma$ were found to decrease by about three orders of magnitude than those of the PPS in air. This result suggests that the absorbed oxygen in PPS plays a role of weak electron acceptor. Secondly, we measured the photocurrent of the annealed PPS under steady-state photoirradiation. The result is shown in Figure 3. The amount of photocurrent was found to be proportional to light intensity and to originate from the dark current in annealed PPS in air.

In PMIA and PEN, one can observe a large photocurrent at low temperatures below $T_{\mathrm{b}}$ in Figure 1. Linear relations between photocurrent and light intensity which originate from the value of dark current at the corresponding temperature were again observable.

In conclusion, it should be emphasized that linear polymers such as PPS, PMIA and PEN, with small $\pi$-conjugated groups but without skeletal delocalized structure exhibit a weak electronic conductivity in dark and a large photoconductivity.

\section{Electronic Conduction in Doped PPS Film}

A theoretical approach has been done on the valence electronic properties in PPS and has afforded the ionization potential, bandwidth and band gap. ${ }^{1}$ This theoretical result suggests that some extents of delocalization occur in PPS and an electronic conductor can be prepared by doping with suitable acceptors. In fact, doping of PPS with a strong inorganic acceptor such as $\mathrm{AsF}_{5}, \mathrm{Br}_{2}$ afforded the electronic conductors with fairly high conductivity. However, these PPS-inorganic acceptor systems remain the following problems to be solved; 1) if the doped PPS film is kept in oxygen or humid atmosphere, it is immediately deteriorated, 2) doping caused change in the chemical structure of PPS. $^{2}$

We have developed a new stable doped system, PPS doped with organic acceptors. Figure 4 illus-

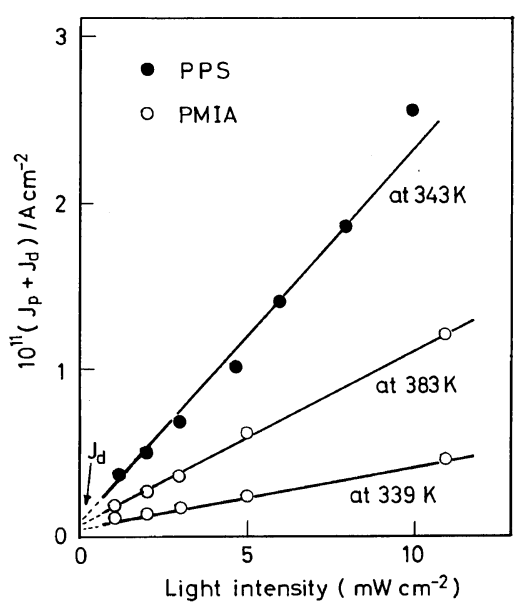

Figure 3. Relation between the amount of steady-state photocurrent and the light intensity.

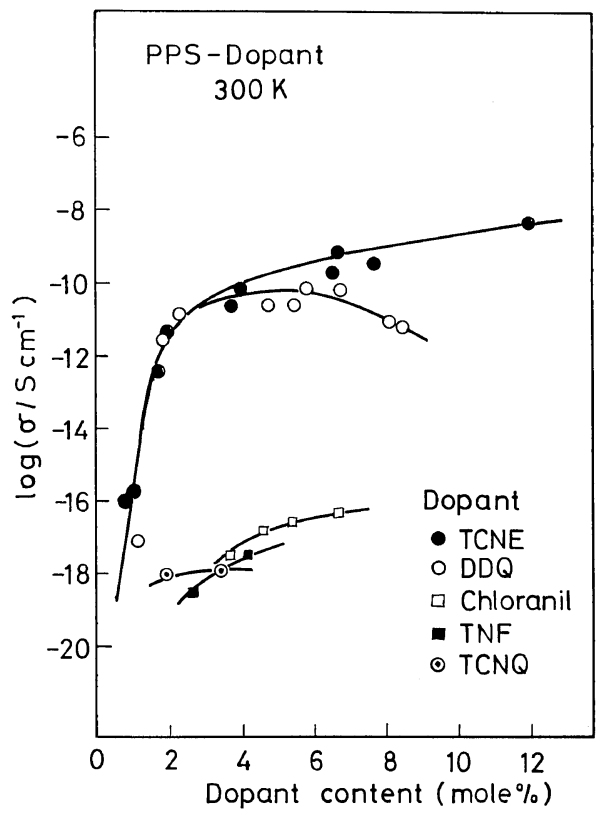

Figure 4. Conductivities at room temperature in doped PPS's as a function of the dopant concentration.

trates the conductivity at room temperature, $\sigma_{\mathrm{RT}}$, as a function of dopant concentration, where the concentration is represented as mole dopants/mole PPS repeat units. Tetracyanoethylene (TCNE) and dichloro-dicyanobenzoquinone (DDQ) were fairly effective for rising of $\sigma_{\mathrm{RT}}$, but chloranil, tetracyanoquinodimethane (TNCQ) and trinitrofluorenone (TNF) were less effective. The difference in 
capability of enhancement of conductivity is considered to be due to differences in the electron affinity and in molecular size. Small molecule with strong electron affinity will cause higher conductivity of the doped system.

PPS films doped with TCNE was found to be tough, flexible, and very stable in air. The relation between $\sigma$ and temperature was reproducible in a couple of succesive measurements in the temperature region below $400 \mathrm{~K}$. After standing of the doped film in air during three months, the same result was obtained.

Temperature dependences of $\sigma$ in PPS films doped with TCNE are shown in Figure 5. Arrhenius relations were valid for the data at temperatures above room temperature. Apparent activation energy for conduction $\Delta E$ was calculated from the slope of the Arrheinius plots. As shown in Figure 5, $\Delta E$ decreases with increase in $\sigma$. Figure 6 indicates the temperature dependences of $\sigma$ in PPS films doped with various dopants, compared at a similar dopant concentration with each other. The similar relation that the higher the conductivity is, the lower the activation energy is, is obtained again. The correlation between $\sigma$ and $\Delta E$ will be discussed later.

In order to understand the conduction mechanism, we should determine the carrier mobility or the carrier density in addition to $\sigma$. We tried to estimate the drift mobility of electronic carriers in

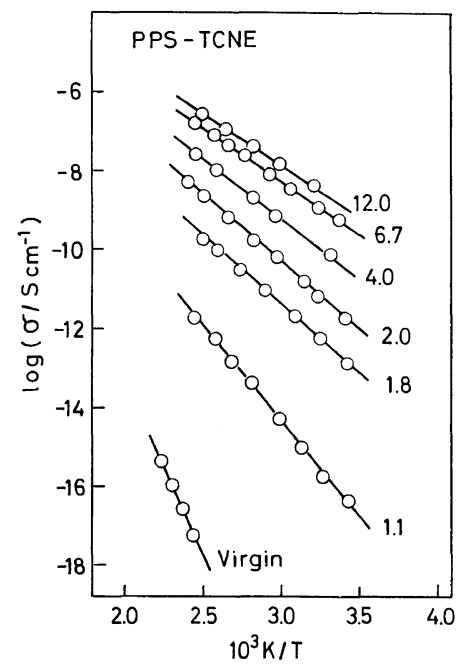

Figure 5. Temperature dependences of the conductivity in PPS films doped with TCNE. the doped PPS films by using the time-of-flight technique. The hole transient currents followed after irradiation of UV light pulse could be observed. The electronic transients were too small to be analysed. The transients revealed that the movement of holes was dispersive. According to Scher and Montroll, ${ }^{3}$ the transit time $t_{\mathrm{T}}$ of carriers in dispersive systems can be determined from $\log I$ (current) vs. $\log t$ (time) plots. Figure 7 is an example for the present sample, though the time axis is represented by reduced time. The intercept of two straight lines which were obtained approximately from the plots in shorter and longer time region

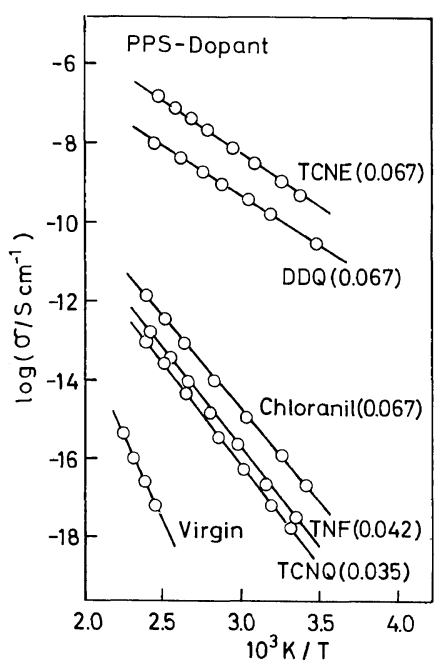

Figure 6. Temperature dependences of the conductivity in PPS films doped with various dopants.

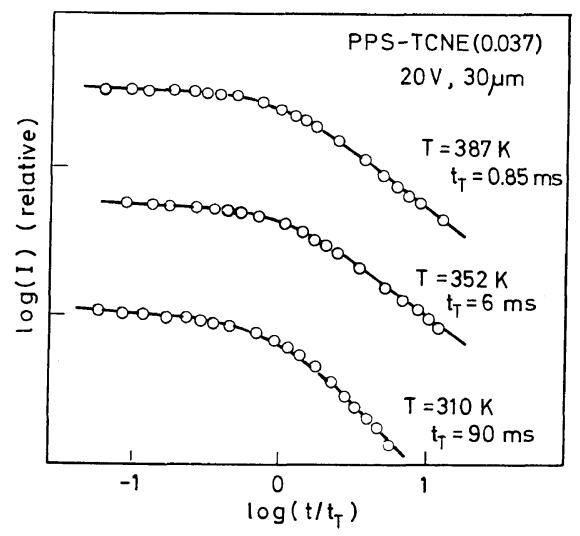

Figure 7. Examples of hole transient current in PPS film doped with TCNE. 


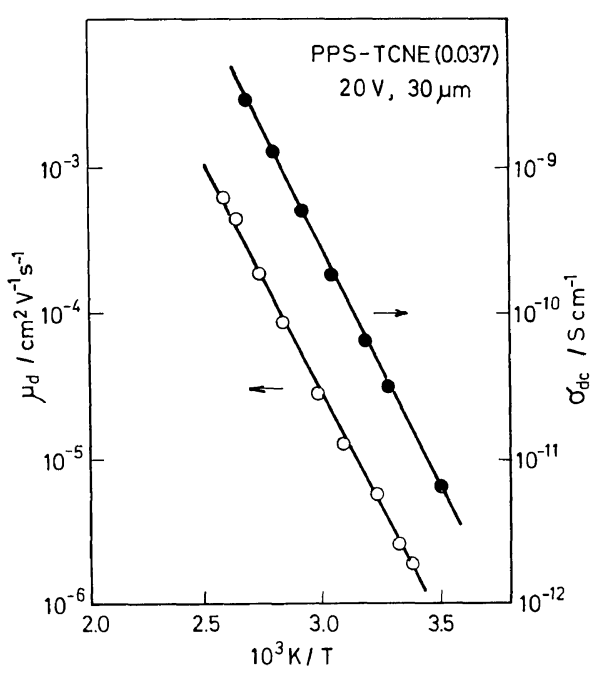

Figure 8. Temperature dependences of the conductivity and the hole mobility in PPS films doped with TCNE.

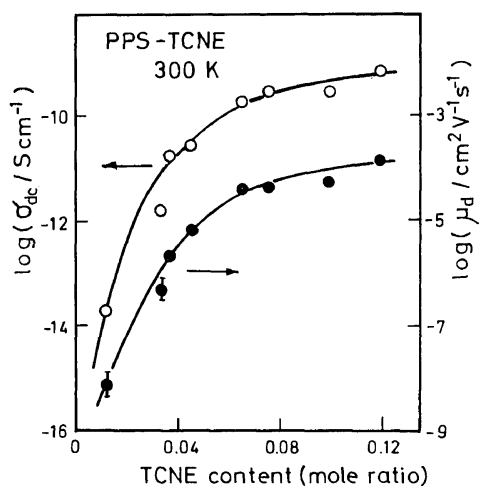

Figure 9. Conductivity and hole mobility at room temperature in the doped PPS films as functions of TCNE content.

gave $t_{\mathrm{T}}$. Since $t_{\mathrm{T}}$ was approximately proportional to $V^{-1}$, we evaluated the hole mobility $\mu$ as $\mu=l^{2} / t_{\mathrm{T}} V$, where $l$ is the sample thickness and $V$ is the applied voltage.

Figure 8 shows the temperature dependences of $\sigma$ and $\mu$ in a PPS-TCNE film. Both slopes of $\log \sigma-$ $T^{-1}$ and $\log \mu-T^{-1}$ coincides with each other and give $\Delta E=0.6 \mathrm{eV}$. The following conclusions can be deduced from the evidence mentioned above.

1. In PPS-TCNE films, the temperature dependence of $\sigma$ is governed by that of $\mu$ and the carrier density is almost independent of tempera-

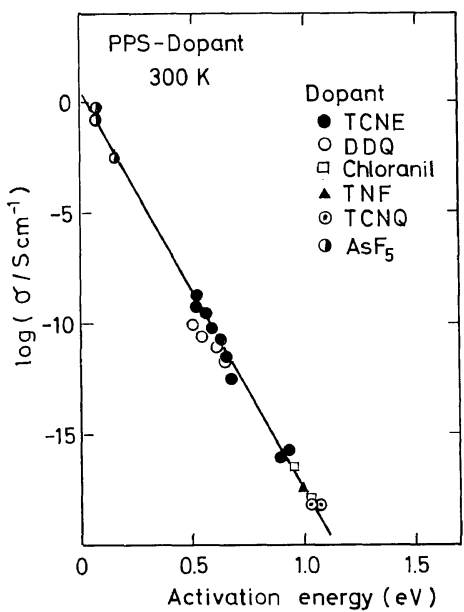

Figure 10. Correlation of the conductivity and the activation energy for conduction in the doped PPS family.

ture.

2. Fairly large value of the activation energy for $\mu$ says that the carrier transport is due to the thermally excited hopping mechanism.

Figure 9 illustrates the variations of $\sigma_{\mathrm{RT}}$ and $\mu_{\mathrm{RT}}$ as functions of TCNE content. Both variations are approximately parallel with each other, at least in the TCNE concentration region above 0.01 . Therefore it is concluded that the principal effect of TCNE doping is enhancement of $\mu$ in this concentration region. This conclusion may be incorrect in the TCNE concentration region from low to zero (undoped). The number of carriers must increase with introduction of a few TCNE dopants at the beginning. Otherwise, unrealistic values for $\mu$ are deduced in those lightly doped and undoped PPS.

An example of the correlation between $\sigma$ and $\Delta E$ in the doped PPS is shown in Figure 10, where the data for PPS- $\mathrm{AsF}_{5}$ system are cited from the $\sigma-T$ relations by Shacklette et al. ${ }^{4}$ in the high temperature region. We have obtained a surprising correlation that the $\log \sigma_{\mathrm{RT}} v s$. $\Delta E$ can be described by a straight line, irrespective of species and concentration of the dopant. The slope of this straight line is approximately equal to $\left(k T_{\mathrm{RT}} \ln 10\right)^{-1}$. These facts say that the following relation is valid in the doped PPS films, that is,

$$
\sigma=K \exp (-\Delta E / k T)
$$

and $K$ is independent of species of the dopant. 
Unfortunately any theoretical analysis can not be developed from above equation, because the chemical structure of PPS is changed in some systems and unchanged in other systems. However, the following concepts would be correct.

1. Carrier transport in all doped PPS films cited in Figure 10 is attributable to the hopping mechanism.

2. The highest level of $\sigma_{\mathrm{RT}}$ which is attainable by the hopping conduction in PPS is approximately $1 \mathrm{~S}$ $\mathrm{cm}^{-1}$.

3. Metal-insulator transition does not occur in the doped PPS films, even in PPS-AsF films. $_{5}$

\section{Electronic Conduction in Other Doped Polymers}

As mentioned above, one can get a picture on the conduction mechanism in PPS systems through the conductivity and mobility studies in terms of dopant concentration and temperature. As to other polymers, it is very important to study the conduction behavior in undoped and.lightly doped systems as well as the highest attainable conductivity. Polypyrrole, polythiophene (PT), poly $(p$ phenylene) (PPP) and poly(2,5-dimethoxy-pphenylene vinylene) $\left(\mathrm{CH}_{3} \mathrm{O}-\mathrm{PPV}\right)$ should be thoroughly investigated, since they are dense films with high stability. If one would investigate the conduction behavior in undoped and doped $\mathrm{CH}_{3} \mathrm{O}-$ PPV, one could observe the metal-insulator transition, because $\sigma$ in this film was found to rise to $10^{3} \mathrm{~S} \mathrm{~cm}^{-1}$.

It should be pointed out that one encounters difficulties in determination of $\sigma$ of the undoped polymers. We have measured the conductivity of PPP which was synthesized from benzene by using Friedel-Crafts catalyst. Figure 11 shows the relation of $\sigma$ at $353 \mathrm{~K}$ to annealing temperature $T_{\mathrm{a}}$. Anealing of PPP in $\mathrm{N}_{2}$ atmosphere is considered to bring about increase of the crystallinity and elimination of halogen which was introduced as residual catalyst. In fact, it was confirmed by a fluorescence $\mathrm{X}$-ray analysis system that the annealing at temperatures above $473 \mathrm{~K}$ resulted a remarkable decrease in chlorine content in PPP. Drastic lowering of $\sigma$ in Figure 11 is considered to be closely connected with the elimination of halogen and with decrease in charge transfer interaction between PPP and halogen. Almost data in Figure 11 should be regarded as those for PPP's doped with halogen. The conductivity of undoped PPP must be lower

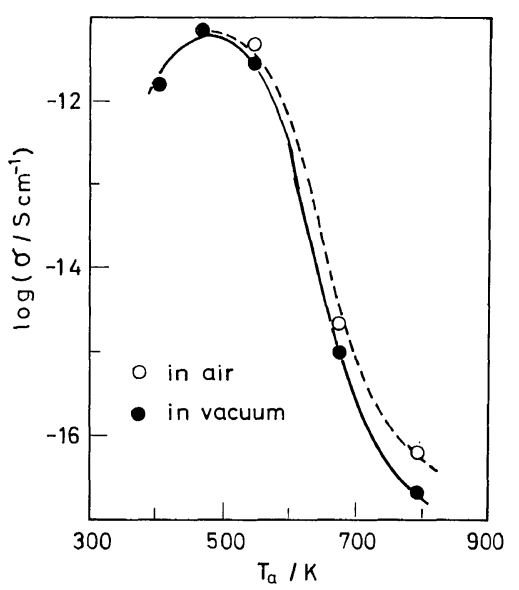

Figure 11. Relation between the conductivity and the annealing temperature of PPP. Open circles, in air; filled circles, in vacuum.

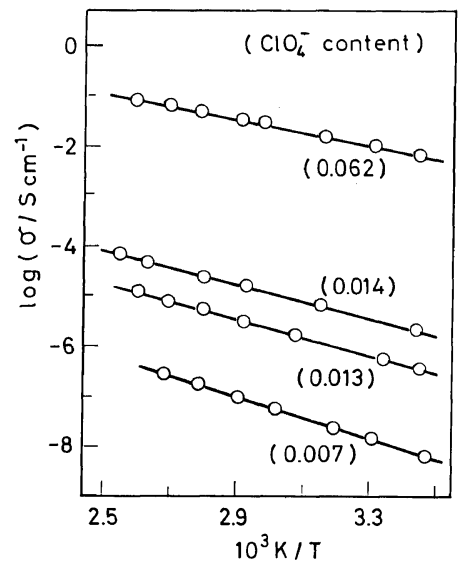

Figure 12. Temperature dependences of the conductivity in PT films doped with $\mathrm{ClO}_{4}{ }^{-}$

than the lowest value in Figure 11 and probably is located at around $10^{-20} \mathrm{~S} \mathrm{~cm}^{-1}$ at room temperature.

Figure 12 shows the temperature dependences of $\sigma$ in PT's with various amounts of dopant $\left(\mathrm{ClO}_{4}{ }^{-}\right)$. With variation of the dopant concentration from 0.062 to $0.007, \sigma_{\mathrm{RT}}$ varies from $10^{-2}$ to $10^{-9} \mathrm{~S} \mathrm{~cm}^{-1}$ and the apparent activation energy for conduction changes from 0.16 to $0.4 \mathrm{eV}$. What is the characteristics of undoped PT? Electrochemical procedure was not sufficient to obtain the undoped PT, because lightly doped PT became an electrical insulator. When the doped PT is kept in $\mathrm{N}_{2}$ atmosphere at high temperatures, one can take away the 


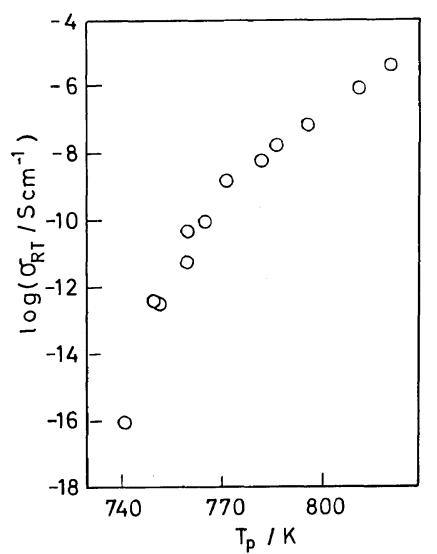

Figure 13. Conductivity at room temperature in lightly pyrolyzed PI as a function of pyrolysis temperature.

role of electron acceptor from $\mathrm{ClO}_{4}{ }^{-}$. The value of $\sigma_{\mathrm{RT}}$ in the annealed PT which was kept at $520 \mathrm{~K}$ for $5 \mathrm{~h}$ was about $10^{-14} \mathrm{~S} \mathrm{~cm}^{-1}$. Probably $\sigma_{\mathrm{RT}}$ in undoped PT must be lower than $10^{-14} \mathrm{~S} \mathrm{~cm}^{-1}$.

\section{Electronic Conduction in Pyrolyzed Polyimides}

Pyrolysis of a suitable polymer, besides the doping with an electron acceptor, is a powerful way for preparation of conducting films with high stability. It has been reported that polyimide pyrolyzed at high temperatures has high conductivity up to $10^{2} \mathrm{~S}$ $\mathrm{cm}^{-1}{ }^{6}$ The structural analysis suggested that polyimide changed into a graphite-like structure with the pyrolysis at very high temperature. ${ }^{7.8}$ If pyrolysis was performed at moderate condition, however, the main structural change in polyimide would be the development of the conjugated double bonds.

Figure 13 shows $\sigma_{\mathrm{RT}}$ in polyimides pyrolyzed at various temperatures. The pyrolysis was took place at a constant temparature in $\mathrm{N}_{2}$ atmosphere for $12 \mathrm{~h}$. As shown in Figure 13, the conductivity was strongly dependent upon the pyrolysis temperature and increased remarkably with progress of the pyrolysis. For instance, $\sigma_{\mathrm{RT}}$ in polyimide pyrolyzed at $743 \mathrm{~K}$ was $10^{-17} \mathrm{~S} \mathrm{~cm}^{-1}$ and that at $833 \mathrm{~K}$ was $10^{-5} \mathrm{~S} \mathrm{~cm}^{-1}$. This fact indicates that the thermal reaction rapidly took place at the pyrolysis temperatures between 743 and $833 \mathrm{~K}$. It was found that a significant weight loss $(10-38 \%)$ occurred by the pyrolysis in this temperature region. The structural change accompanied by the weight loss is con-

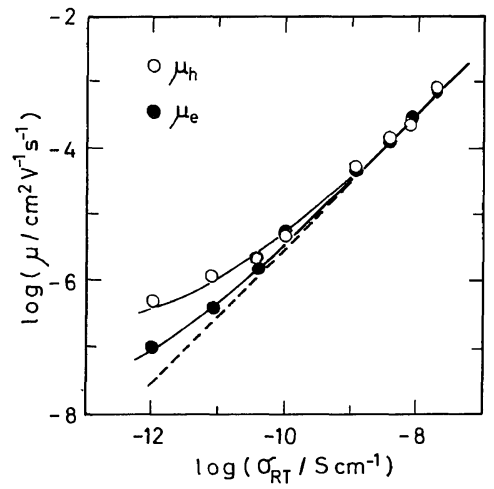

Figure 14. Relationship between the conductivity and the carrier mobilities in lightly pyrolyzed PI films.

sidered to cause the increase of $\sigma_{\mathrm{RT}}$. A spectroscopic study of the pyrolyzed polyimide showed development of the conjugated double bonds; for example, the absorption peak at $355 \mathrm{~nm}$ for polyimide pyrolyzed at $763 \mathrm{~K}$ shifted toward longer wavelength in polyimide pyrolyzed at a higher temperature. Therefore, the increase of the conductivity in lightly pyrolyzed polyimide is attributable to the development of the conjugated double bonds.

The carrier mobility of the pyrolyzed polyimide was determined by the time-of-flight technique. The shapes of the transient photocurrent revealed that charge transport process was dispersive, like in PPSorganic dopant films. By the similar procedure to that in the case of PPS systems, we have determined the transit time $t_{\mathrm{T}}$ and the drift mobility $\mu$. Figure 14 illustrates the relationship between the carrier mobility and the conductivity in pyrolyzed polyimide, where $\mu_{\mathrm{h}}$ and $\mu_{\mathrm{e}}$ denote the mobility of hole and electron respectively. The conductivity is nearly proportional to $\mu$ in the $\sigma_{\mathrm{RT}}$ range from $10^{-12}$ to $10^{-8} \mathrm{~S} \mathrm{~cm}^{-1}$. The dotted line in Figure 14 is a straight line with slope 1 . Deviations from this straight line are observed in low mobility and low conductivity regions, as shown in Figure 14. These evidences suggest that the carrier density increases at the beginning stage of the pyrolysis and then the increase of $\mu$ becomes main factor for enhancement of $\sigma$ with progress of pyrolysis.

We have also confirmed that a parallel relation between the activation energy of conductivity and that for the mobility; the value ranges from 0.4 to $0.2 \mathrm{eV}$ in polyimide pyrolyzed at temperature from 753 to $793 \mathrm{~K}$. A fairly large value of the activation 
energy for carrier transport means that the conduction is due to a hopping mechanism.

The carrier density of the polyimide pyrolyzed at $783 \mathrm{~K}$ was estimated to be $10^{14} \mathrm{~cm}^{-3}$ from the value of the conductivity and the mobility. On the other hand, Brom et al. measured the Hall effect and estimated that the carrier density in pyrolyzed polyimide was $10^{21} \mathrm{~cm}^{-3}{ }^{6}$ Their polyimide was pyrolyzed at $1023 \mathrm{~K}$ which was about $250 \mathrm{~K}$ higher than in our case. The difference in the carrier density is considered to result from the difference in the pyrolysis reaction, that is, a graphite-like structure is formed or not.

\section{CONCLUSION}

We have performed the experimental study on the conduction behavior in some polymers, with the aims of better understanding of the conduction mechanism and of obtaining the fundamental informations for design of stable electron-conducting polymers. The conclusions are as follows;

1. Linear polymers, such as PPS, PMIA, and PEN, with small $\pi$-conjugated groups but without skeletal delocalized structure exhibit a weak electronic conductivity, even in the undoped state.

2. The conductivity in PPS can be varied in the range from $10^{-20}$ to $10^{-9} \mathrm{~S} \mathrm{~cm}^{-1}$ by the doping with organic electron acceptors.

3. The main effect of the doping of PPS is enhancement of the carrier mobility, though the doping at the beginning stage causes an increase of the carrier density.

4. In PPS family, a simple relation between the conductivity and the activation energy for con- duction is valid irrespective of species and concentration of the dopant. Judging from this relation, the metal-insulator transition does not occur in the doped PPS films, even in PPS-AsF ${ }_{5}$.

5. There remain some problems in evaluation of the conduction behavior in undoped polymers, such as undoped PPS, PPP, and PT.

6. Light pyrolysis of polyimide is useful too for obtaining a variety of electron-conducting films, if it is well controlled. Enhancement of the conductivity in lightly pyrolyzed polyimide is attributed to the devlopment of the conjugated double bonds and closely connected with the increase of the carrier mobility.

7. In all systems studied here, the carrier transport is due to the hopping mechanism and the metal-insulator transition does not occur.

\section{REFERENCES}

1. J. L. Brédas, R. L. Elsenbaumer, R. R. Chance, and R. Silbey, J. Chem. Phys., 78, 5656 (1983).

2. R. H. Baughman, J. L. Brédas, R. R. Chance, R. L. Elsenbaumer, and L. W. Shacklette, Chem. Rev., 82, 209 (1982).

3. H. Scher and E. W. Montroll, Phys. Rev., B12, 2455 (1975).

4. L. W. Shacklette, R. L. Elsenbaumer, R. R. Chance, H. Eckhardt, J. E. Frommer, and R. H. Baughman, J. Chem. Phys., 75, 1919 (1981).

5. M. Hirooka, private communication.

6. H. B. Brom, Y. Tomkiewicz, A. Aviram, A. Broers, and B. Sunners, Solid State Commun., 35, 135 (1980).

7. J. I. Gittleman and E. K. Sichel, J. Electr. Materials, 10, 327 (1981).

8. E. K. Sichel and T. Emma, Solid State Commun., 41, 747 (1982). 